\title{
Kawasaki disease complicated by peripheral gangrene and a ventricular septal defect: An unusual association
}

\author{
Muhammad Mohsin, Saleem Akhtar \\ Division of Pediatric Cardiology, Department of Pediatrics, Aga Khan University Hospital, Karachi, Pakistan. \\ E-mail: mohsin217529@gmail.com \\ Received: 17th September 2017, Revised: 31st December 2017, Accepted: 1st January 2018
}

\begin{abstract}
SUMMARY: Mohsin M, Akhtar S. Kawasaki disease complicated by peripheral gangrene and a ventricular septal defect: An unusual association. Turk J Pediatr 2019; 61: 301-304.
\end{abstract}

Kawasaki disease is an acute febrile illness associated with the vasculitis of small and medium size arteries. Peripheral gangrene is very rare but a known complication of Kawasaki disease mostly reported in the infantile age group. Early therapy is advocated for prevention of serious complications. We report a 10-month-old girl with Kawasaki disease and a small ventricular septal defect who developed peripheral gangrene despite initiation of early therapy.

Key words: infant, complications, Kawasaki disease, peripheral gangrene.

Kawasaki disease (KD) is an acute illness of unknown cause that chiefly affects infants and children and is characterized especially by fever, rash, conjunctivitis, inflammation of lips and tongue, and unilateral swollen lymph nodes of the neck and is the leading cause of acquired heart disease. ${ }^{1-3} \mathrm{KD}$ is associated with systemic vasculitis particularly affecting the coronary arteries, causing coronary artery aneurysms (CAA) in $15-25 \%$ of untreated patients while $2-3 \%$ of untreated cases die as a result of coronary vasculitis. ${ }^{4-6}$ Peripheral gangrene is a known but rare complication of KD that occurs almost exclusively in infants. ${ }^{7-15}$ Treatment with intravenous immunoglobulin (IVIG) before day 10 of illness has been shown to reduce the incidence of coronary artery aneurysm. ${ }^{4}$ It is speculated that early treatment might also reduce the incidence of peripheral ischemia and gangrene. ${ }^{10}$

In this report, we describe a recent case of infantile KD and ventricle septal defect associated with peripheral gangrene, in which the infant had been treated with IVIG at the earliest date of disease onset but still developed peripheral gangrene. This is the first such case to be reported from Pakistan.

\section{Case Report}

A 10-month-old girl presented to the emergency department with history of fever for 5 days associated with a maculopapular rash, irritability, strawberry tongue, conjunctivitis and edema of the hands and feet. The fever was high grade, intermittent and associated with irritability. Four days later, the child developed a maculopapular rash which was generalized. It was associated with redness of the oral cavity and conjunctivitis. On examination, the child had a length of $75 \mathrm{~cm}$ and weight of $8.5 \mathrm{~kg}$. She was irritable, febrile (101 F) with a heart rate of $120 / \mathrm{min}$ and blood pressure of $96 / 53 \mathrm{~mm}$ hg while maintaining oxygen saturation at room air. Generalized maculopapular rash was present with pallor and bilateral pedal and dorsal edema. The patient had a strawberry tongue and bilateral nonpurulent conjunctivitis. Systemic examination did not reveal any other positive signs. The laboratory examinations were as follows: $\mathrm{Hb}$ $8.1 \mathrm{~g} / \mathrm{dl}$; WBC $32,100 / \mathrm{mm}^{3}$; platelet 2,91,000/ $\mathrm{mm}^{3}$; ESR $81 \mathrm{~mm} / \mathrm{h}$; CRP $19.5 \mathrm{mg} / \mathrm{dl}$; Albumin $3 \mathrm{~g} / \mathrm{dl}$ and SGPT $70 \mathrm{IU} / \mathrm{L}$. The child was admitted to the pediatric intensive care unit (PICU) and broad spectrum antibiotics were started. Echocardiogram showed a small 2 $\mathrm{mm}$ perimembranous ventricular septal defect (VSD) covered with tricuspid aneurysmal tissue and normal right coronary artery measuring 1.8 $\mathrm{mm}$ at origin with $\mathrm{Z}$ score value of +0.76 and a dilated left main coronary artery measuring 


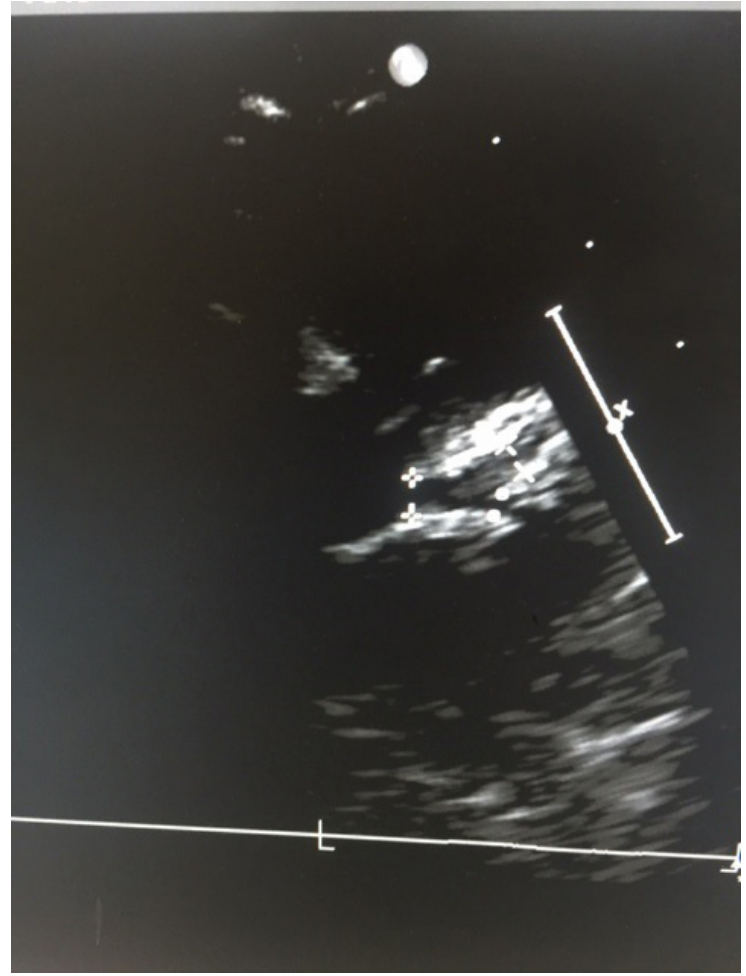

Fig. 1. Left coronary artery dilatation

$3.0 \mathrm{~mm}$ at ostium with $\mathrm{Z}$ score value of +2.61 and $2.8 \mathrm{~mm}$ at bifurcation, LAD measuring 2.3-2.4 $\mathrm{mm}$ with $\mathrm{Z}$ score value of +3.02 (Fig. $1)$. No convincing coronary artery aneurysm was found. Under a diagnosis of $\mathrm{KD}$, high-dose IVIG $(2 \mathrm{~g} / \mathrm{kg}$, continuous intravenous infusion for 12 hours) and oral aspirin $(100 \mathrm{mg} / \mathrm{kg} /$ day $)$ were administered. On the $7^{\text {th }}$ day of illness the child developed discoloration of left big toe (Fig. 2). Ultrasound Doppler showed normal flow in the left dorsalis pedis and posterior tibial arteries. Anticoagulation with low molecular weight heparin and local vasodilation with topical nitroglycerine paste was started after consultation with vascular surgery and monitoring for extension of gangrene was continued. On day 10 of the illness, it was well demarcated and only confined to the left big toe. Repeat echocardiogram on $14^{\text {th }}$ day of illness ruled out coronary artery aneurysm. Fever subsides after the $1^{\text {st }}$ dose of IVIG and further evaluation revealed a decreased ESR, CRP, total WBC and increasing platelet count. The patient was discharged home on low molecular weight heparin and aspirin in a stable condition.

During the course of illness she continued to

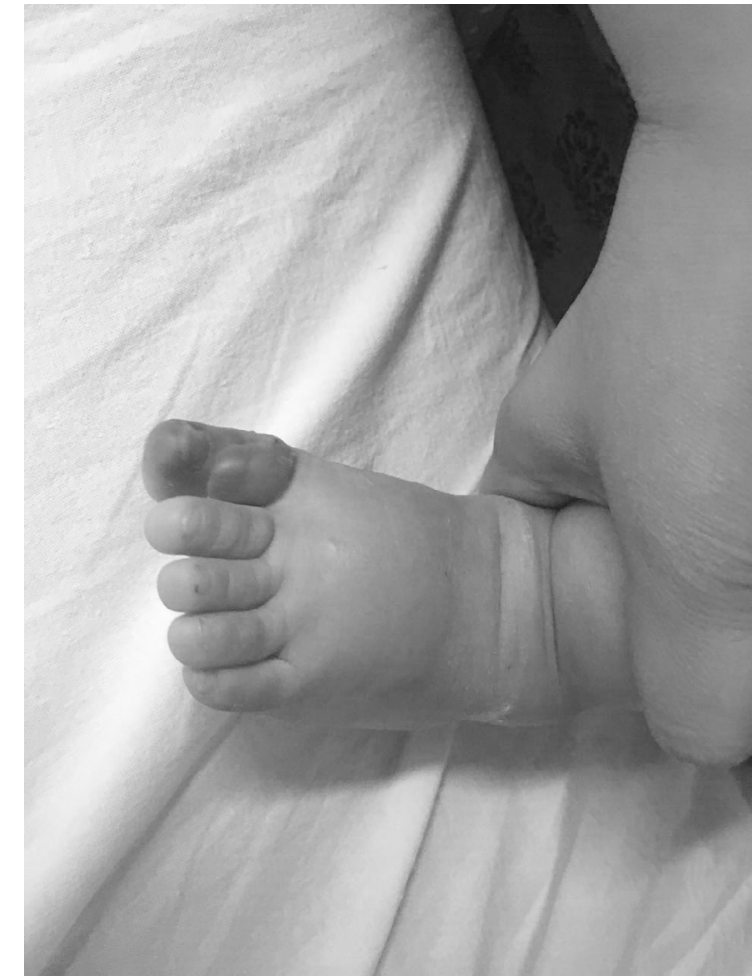

Fig. 2. Gangrene of left big toe

develop other features of peripheral vasculitis such as changes of the skin, changes on the BCG scar (Fig. 3) and changes on the eyelids (Fig. 4).

A written informed consent was taken from parents before submission of this case report.

\section{Discussion}

Kawasaki disease (KD) is an acute selflimiting inflammatory disorder, associated with vasculitis, affecting predominantly small to medium-sized arteries, particularly the coronary arteries. Very few cases of KD with peripheral gangrene and auto amputation have been reported in the literature.

Although the pathogenesis of this complication is not well understood, it likely includes some combination of local peripheral arteritis, arteriospasm, thrombosis peripherally and/ or more proximally (e.g., in an axillary artery aneurysm), and cardiogenic shock. ${ }^{16}$ The previous cases of KD complicated with peripheral gangrene were infants except one adult patient. ${ }^{17}$ Our patient was also an infant. The possible explanation of peripheral gangrene with $\mathrm{KD}$ in the infantile age group 


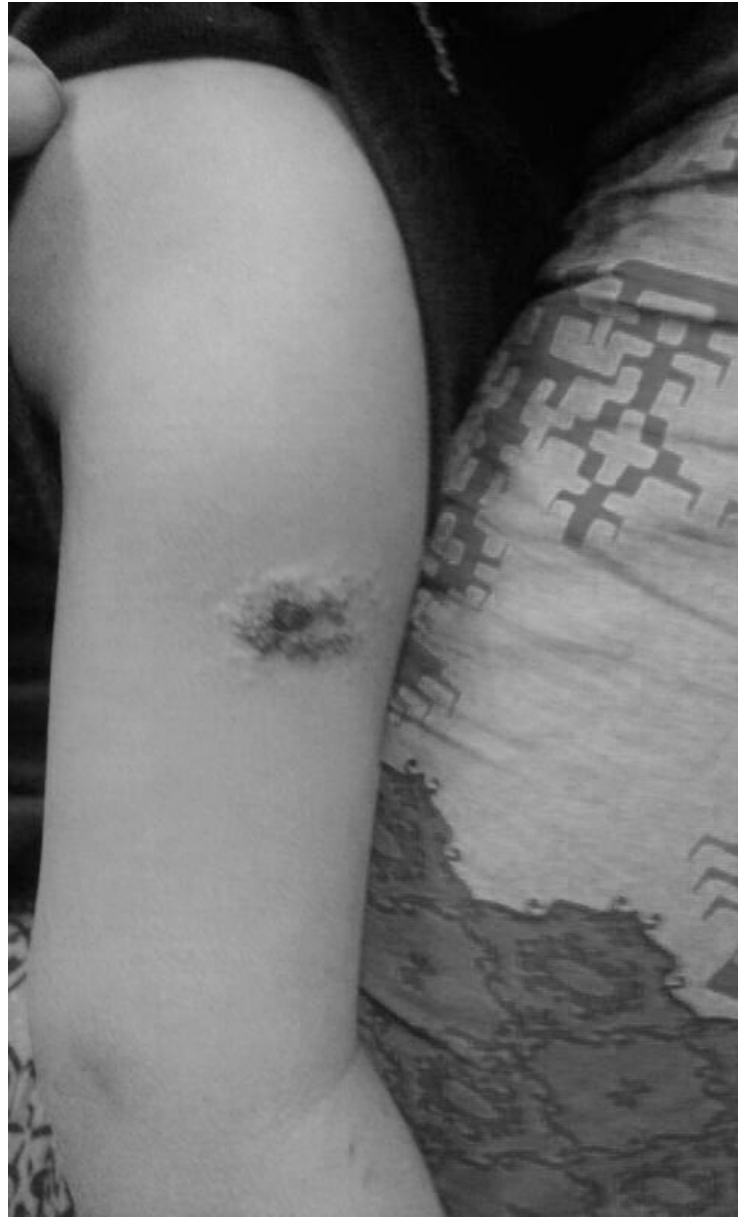

Fig. 3. Changes in BCG scar

is delayed diagnosis and treatment due to atypical presentation in this age group, but in our patient treatment was initiated early. To our knowledge the association of VSD with $\mathrm{KD}$ complicated by peripheral gangrene has never been reported in the literature.

Currently, there is no consensus regarding the ideal therapy for peripheral ischemia in the aforementioned case reports. There is a wide range of different medications which are used in the management of KD with gangrene. Some of the treatment agents that have been used are heparin, steroids, hydralazine, propranolol, warfarin, urokinase, Prostaglandin E1, dipyridamole, nitroprusside, tissue plasminogen activator, prostacyclin, glyceryl trinitrate, nifedipin, and even caudal block. ${ }^{8}$ However, von Planta et al. ${ }^{17}$ cited the concern of stealing perfusion from potentially compromised coronary arteries as their patient developed a myocardial infarction shortly

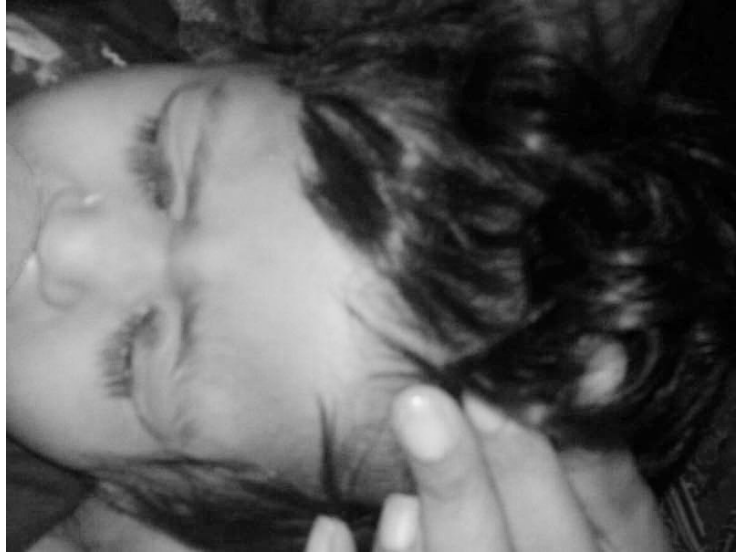

Fig. 4. Changes of eyelids

after the initiation of prostaglandin infusion. In our case, it appeared that IVIG and high dose aspirin were helpful in controlling the inflammation, as fever resolved soon after initiation of these therapies along with low molecular weight heparin for peripheral gangrene. The risk/benefit with prostaglandin infusion needs to be further evaluated.

\section{REFERENCES}

1. Ames EL, Jones JS, Van Dommelen B, Posch JL. Bilateral hand necrosis in Kawasaki syndrome. J Hand Surg Am 1985; 10: 391-395.

2. Belay ED, Maddox RA, Holman RC, Curns AT, Ballah K, Schonberger LB. Kawasaki syndrome and risk factors for coronary artery abnormalities: United States, 1994-2003. Pediatr Infect Dis J 2006; 25: 245-249.

3. Bonté I, Mahr A, Laroche L, Guillevin L, Robineau M. Peripheral gangrene in adult-onset Kawasaki disease. Scand J Rheumatol 2005; 34: 71-73.

4. Brenner JL, Jadavji T, Pinto A, Trevenen C, Patton D. Severe Kawasaki disease in infants: two fatal cases. Can J Cardiol 2000; 16: 1017-1023.

5. Brogan PA, Bose A, Burgner D, et al. Kawasaki disease: an evidence based approach to diagnosis, treatment, and proposals for future research. Arch Dis Child 2002; 86: 286-290.

6. Chang J-S, Lin J-S, Peng C-T, Tsai C-H. Kawasaki disease complicated by peripheral gangrene. Pediatr Cardiol 1999; 20: 139-142.

7. Dillon MJ, Eleftheriou D, Brogan PA. Medium-sizevessel vasculitis. Pediatr Nephrol 2010; 25: 1641-1652.

8. Durall AL, Phillips JR, Weisse ME, Mullett CJ. Infantile kawasaki disease and peripheral gangrene. Pediatr Crit Care Med 2004; 5: 511.

(AAP Abstracts)

9. Gardner-Medwin JM, Dolezalova P, Cummins C, Southwood TR. Incidence of Henoch-Schonlein purpura, Kawasaki disease, and rare vasculitides in children of different ethnic origins. Lancet 2002; 360: 1197-1202. 
10. Harnden A, Alves B, Sheikh A. Rising incidence of Kawasaki disease in England: analysis of hospital admission data. BMJ 2002; 324: 1424-1425.

11. Krohn C, Till H, Haraida S, et al. Multiple intestinal stenoses and peripheral gangrene: a combination of two rare surgical complications in a child with Kawasaki disease. J Pediatr Surg 2001; 36: 651-653.

12. Newburger JW, Takahashi M, Gerber MA, et al; Committee on Rheumatic Fever, Endocarditis and Kawasaki Disease; Council on Cardiovascular Disease in the Young; American Heart Association; American Academy of Pediatrics. Diagnosis, treatment, and longterm management of Kawasaki disease. A statement for health professionals from the Committee on Rheumatic Fever, Endocarditis and Kawasaki Disease, Council on Cardiovascular Disease in the Young, American Heart Association. Circulation 2004; 110: 2747-2771.
13. Teixeira $\mathrm{OH}$, Pong $\mathrm{AH}$, Vlad P. Amputating gangrene in Kawasaki disease. Can Med Assoc J 1982; 127 : 132-134.

14. Tomita S, Chung K, Mas M, Gidding S, Shulman ST Peripheral gangrene associated with Kawasaki disease. Clin Infect Dis 1992; 14: 121-126.

15. Trumble T, Fitch RD. Kawasaki disease: A cause of vasculitis in children. J Pediatr Orthop 1986; 6: 92-95.

16. Westphalen MA, McGrath MA, Kelly W, Moore FJ, Ziegler JB. Kawasaki disease with severe peripheral ischemia: treatment with prostaglandin E1 infusion. J Pediatr 1988; 112: 431-433.

17. von Planta M, Fasnacht M, Holm C, Fanconi S, Seger R. Atypical Kawasaki disease with peripheral gangrene and myocardial infarction: therapeutic implications. Eur J Pediatr 1995; 154: 830-834. 